Artigo Original

\title{
Pontos de transição da frequência cardíaca em teste progressivo máximo ${ }^{1}$
}

\author{
Patrícia Guimarães Couto ${ }^{1}$ \\ Ana Paula Rodrigues ${ }^{2}$ \\ Antônio José Ferreira Júnior ${ }^{3}$ \\ Sandro Fernandes da Silva ${ }^{2}$ \\ Fernando Roberto de-Oliveira ${ }^{2}$ \\ ${ }^{1}$ Programa de Pós-graduação em Educação Física e Esporte, Universidade de São Paulo, SP, Brasil \\ ${ }_{2}^{2}$ Departamento de Educação Física, Universidade Federal de Lavras, Lavras, MG, Brasil \\ ${ }^{3}$ Programa de Pós-Graduação em Educação Física, Universidade Federal de Juiz de Fora, Juiz \\ de Fora, MG, Brasil
}

\begin{abstract}
Resumo: Foi realizada análise do comportamento da frequência cardíaca $(F C)$ e identificação dos pontos de inflexão (PIFC) e de deflexão da FC (PDFC) em teste progressivo máximo, em sujeitos do sexo feminino e masculino. Vinte universitários foram submetidos ao teste em cicloergômetro. A FC foi monitorada para posterior análise e identificação dos pontos de transição (PT). A FC apresentou comportamento sigmóide, com identificação de PT em todos os sujeitos, sendo: a) em 65\% PIFC (64 $\pm 27 \mathrm{~W} ; 29 \pm 9 \%$ Pmáx e $126 \pm$ 12bpm; $66 \pm 5 \% F C m a ́ x)$ e PDFC (177 $\pm 45 \mathrm{~W} ; 81 \pm 10 \%$ Pmáx e $178 \pm 8 \mathrm{bpm}$; $93 \pm 4 \% F C$ máx); b) em 30\% apenas PIFC (80 $\pm 32 \mathrm{~W} ; 36 \pm 14 \%$ Pmáx e $125 \pm 13 \mathrm{bpm} ; 66 \pm 5 \%$ FCmáx) e c) em $5 \%$ o PDFC isolado (103W; 57\%Pmáx e 150bpm; 82\%FCmáx). O PIFC foi encontrado em carga significativamente inferior ao PDFC, sem diferenças na carga e FC relativas entre os sexos.
\end{abstract}

Palavras-chave: Exercício. Aptidão Física. Modulação Autonômica.

\section{Transition points of heart rate in maximum progressive test}

\begin{abstract}
Was analyzed the Heart Rate (HR) behavior and identified the HR inflection points (HRIP) and deflection points (HRDP) in a maximum progressive test, using female and male subjects. Twenty university students were submitted to a maximum progressive test. The HR was monitored to posterior analysis and identification of the transition points (TP). The subjects' HR showed sigmoid behavior, with TP identification in all subjects, being: a) in 65\% HRIP (64 $\pm 27 \mathrm{~W} ; 29 \pm 9 \%$ Pmax and $126 \pm 12 \mathrm{bpm} ; 66 \pm 5 \% \mathrm{HRmax}$ ) and HRDP (177 $\pm 45 \mathrm{~W} ; 81 \pm 10 \%$ Pmax and $178 \pm 8 \mathrm{bpm}$; $93 \pm 4 \% \mathrm{Hrmax}) ; \mathrm{b})$ in $30 \%$ only the HRIP (80 $\pm 32 \mathrm{~W}$; $36 \pm 14 \%$ Pmax and $125 \pm 13 \mathrm{bpm}$; $66 \pm 5 \% \mathrm{HRmax}$ ) and c) in 5\% HRDP isolated (103W; 57\%Pmax and $150 \mathrm{bpm} ; 82 \%$ HRmax). The HRIP was found in significantly lower load than HRDP and there were no differences in the load and HR relative between genders.
\end{abstract}

Keywords: Exercise. Physical Fitness. Autonomic Modulation.

\section{Introdução}

A curva da frequência cardíaca (FC) durante esforço em cargas progressivas possui, na maioria das vezes, característica sigmóide, ou seja, é possível identificar três distintas fases sequenciais: uma fase antecipatória, uma linear em que a inclinação da FC é consistente - e uma fase curvilinear - em que a inclinação da FC em relação à carga de trabalho tem um aumento menos pronunciado (BROOKE; HAMLEY, 1972).

Observando a curva da FC em teste com incrementos de velocidade a cada 200 metros de

\footnotetext{
${ }^{1}$ Resumo apresentado anteriormente no VI Congresso Internacional de Educação Física e Motricidade Humana e XII Simpósio Paulista de Educação Física, realizado em Rio Claro-SP/2009, publicado na Motriz - Revista de Educação Física - UNESP, 2009. v. 15. p. 409.
}

corrida percorridos, Conconi et al., (1982; 1996) sugeriram que era possível identificar um ponto de quebra da linearidade da FC em cargas próximas à velocidade máxima, e que este se relacionava com o segundo limiar de lactato $\left(\mathrm{LL}_{2}\right)$. Este ponto de quebra foi então denominado de ponto de deflexão da FC (PDFC) e vem sendo utilizado como método alternativo para predizer o limiar anaeróbio. Desde então, inúmeros estudos vem sendo conduzidos com este propósito (RIBEIRO et al., 1985; HOFMANN et al., 1994; BUNC et al., 1995; De-OLIVEIRA, 2004; CARMINATTI, 2006).

Observando o comportamento das distintas porções da curva FC $x$ intensidade de esforço, Lima (1997) propôs a identificação do primeiro 
ponto de quebra da $\mathrm{FC}$, que corresponderia então à primeira mudança de direção da $\mathrm{FC}$ na curva de desempenho, e denominou este de ponto de inflexão da FC (PIFC). Cambri et al., (2006), verificaram que em teste progressivo máximo em esteira ergométrica é possível identificar os dois PT, embora nem sempre em conjunto. Assim, em curvas de FC e cargas progressivas de trabalho é possível identificar dois pontos de mudança de direção, que correspondem aos pontos de transição (PT) metabólica durante o esforço (SKINNER; McLELLAN, 1980).

Tendo em vista que este método parece ser uma metodologia alternativa de natureza não invasiva para a identificação dos PT metabólica, comumente utilizados para avaliação aeróbia e prescrição/controle da intensidade de treinamento, é necessária melhor descrição desses PT para verificar sua aplicabilidade e consistência de identificação.

Nesse sentido, foi sugerido também que o PDFC é mais facilmente identificado em sujeitos com maior nível de condicionamento físico (LUCIA et al., 1999), devido ao maior volume de ejeção e maior fração de ejeção ventricular esquerda nesses sujeitos, possibilitando um platô na FC em cargas superiores comparados a sujeitos sedentários. Tendo em vista que essas variáveis (volume de ejeção e fração de ejeção do ventrículo esquerdo) afetam o comportamento cardiovascular em teste incremental (BERNARD et al., 1997; HAWKINS et al., 2007), elas podem consequentemente afetar a identificação dos PT. Dessa forma, parece razoável imaginar que os PT sejam encontrados em cargas absolutas superiores nos homens que nas mulheres, uma vez que estas apresentam menor volume sistólico, menor dimensão do ventrículo esquerdo e menor concentração de hemoglobina, quando comparado aos homens de mesma idade (WILMORE; COSTILL, 1994; FOX et al., 1998; SURAWICZ; PARIKN, 2003), sendo necessária a verificação de diferenças na identificação do PIFC e PDFC entre homens e mulheres, em decorrência dessas alterações cardíacas inerentes ao gênero.

O objetivo deste estudo foi analisar 0 comportamento da FC e identificar os PT da FC em teste progressivo máximo no cicloergômetro, comparando sujeitos do sexo feminino e masculino.

\section{Material e métodos}

Amostra

Foram avaliados vinte estudantes de graduação do curso de Educação Física, saudáveis e não atletas, dez do sexo masculino $(20,9 \pm 1,73$ anos, $81,29 \pm 12,58 \mathrm{Kg}, 1,77 \pm 0,05$ $\mathrm{m}, 20,1 \pm 9,2 \% \mathrm{G})$ e dez do sexo feminino (20,4 \pm 1,07 anos, $61,23 \pm 9,4 \mathrm{Kg}, 1,63 \pm 0,06 \mathrm{~m}, 22,3 \pm$ $7,52 \% \mathrm{G}$ ). Os estudantes participaram voluntariamente do estudo e assinaram um Termo de Consentimento Livre e Esclarecido, aprovado pelo comitê de ética da Universidade de Itaúna (n- 010/07) e de acordo com a Declaração de Helsink (ASSOCIACÃO MÉDICA MUNDIAL, 1989).

\section{Coleta de dados}

Foram feitas indicações prévias para os sujeitos quanto aos hábitos adotados no período anterior ao teste, como não ingerir álcool e café nas 24 horas antecedentes, além de não consumir alimentos por duas horas antes da sua realização, bem como não fazer exercícios físicos extenuantes no dia precedente.

Todos os testes foram realizados no Laboratório de Estudos do Movimento Humano LEMOH - da Universidade Federal de Lavras, no período vespertino. As medidas antropométricas foram mensuradas antes do início dos testes, sendo: estatura (estadiômetro Asimed ${ }^{\circledR}$, Espanha), massa corporal (balança portátil Britânia $^{\circledR}$, Brasil) e dobras cutâneas das regiões do abdômen, tríceps e subescapular (adipômetro Sanny ${ }^{\circledR}$, Brasil), conforme a padronização do protocolo de três dobras, proposto por Lohman (1981).

O teste progressivo máximo (TPM) foi realizado em cicloergômetro (Ergo FIT ${ }^{\circledR}$ 167, Alemanha). Os sujeitos fizeram aquecimento de três minutos a 15 watts $(\mathrm{W})$. Após o aquecimento foi realizado o TPM, com carga inicial de $15 \mathrm{~W}$ e incrementos de $15 \mathrm{~W}$ a cada minuto, até que o avaliado atingisse a exaustão voluntária ou não conseguisse manter a cadência requerida (60 a 70 rpm). Em termos práticos, são necessárias baixas cargas iniciais e pequenos incrementos para a identificação dos dois PT, para uma análise mais completa do comportamento da FC em função do incremento da intensidade de trabalho (LIMA, 1997).

A FC foi coletada ao final de cada estágio, utilizando o cardiofrequêncimetro Polar $^{\circledR}$ (S810i, 
Finlândia). Para que $O$ teste fosse considerado máximo os sujeitos deveriam atingir pelo menos $90 \%$ da FCmáx predita (220 - idade). Para a análise do comportamento da FC utilizamos o programa Graphpad Prism, versão 5.00 (2007 Graphpad Software Incorporated ${ }^{\circledR}$ ).

Os dados de FC e carga foram plotados a cada minuto para que fossem identificados os PIFC e PDFC através do modelo matemático de Cambri et al. (2006). Neste modelo, todos os valores FC - carga são ajustados por uma função polinomial de terceiro grau e por uma equação linear de primeiro grau derivadas dos dados de cada um dos sujeitos (Figura 1). Para análise dos dados foi utilizado o software statistica 7.0.

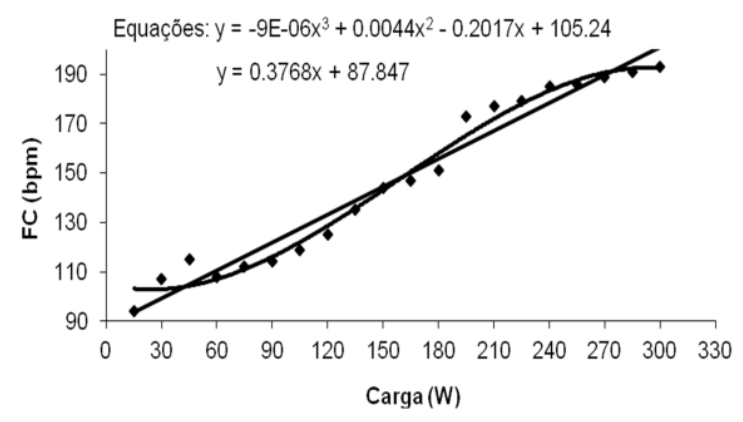

Figura 1. Valores da frequência cardíaca (FC) $\mathrm{x}$ carga, ajustados por equação polinomial de terceiro grau e equação linear de primeiro grau. Exemplo de um indivíduo.

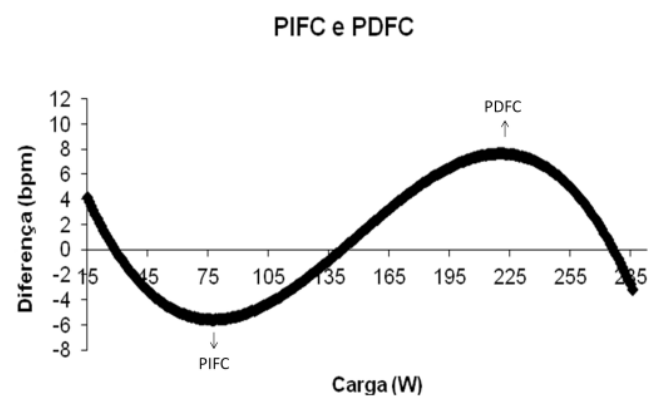

Figura 2. Curva da diferença dos valores de frequência cardíaca $(F C)$ obtidos por equação polinomial de terceiro grau e equação linear de primeiro grau, na qual foram observados o ponto de inflexão da FC (PIFC) e ponto de deflexão da FC (PDFC). Exemplo de um indivíduo.

Após, foi calculada a diferença dos valores de FC obtidos pelas respectivas equações, e projetada uma curva com estes valores. O PIFC foi identificado como o menor valor encontrado da diferença dos valores de FC obtidos pelas equações e o PDFC como o maior valor encontrado, antes da mudança na direção da curva (Figuras 2 - 4)

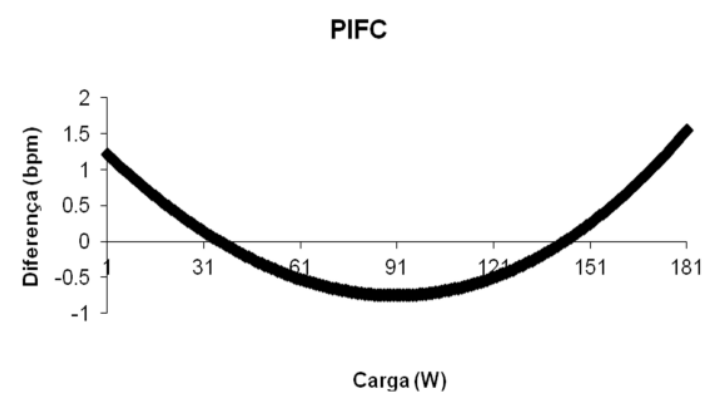

Figura 3. Curva da diferença dos valores de frequência cardíaca $(F C)$ obtidos por equação polinomial de terceiro grau e equação linear de primeiro grau, na qual foi observado apenas o ponto de inflexão da FC (PIFC). Exemplo de um indivíduo.

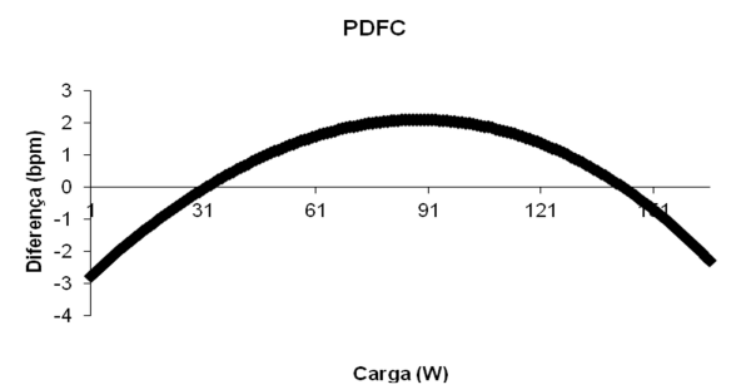

Figura 4. Curva da diferença dos valores de frequência cardíaca $(F C)$ obtidos por equação polinomial de terceiro grau e equação linear de primeiro grau, na qual foi observado somente o ponto de deflexão da FC (PDFC). Exemplo de um indivíduo.

\section{Análise estatística}

Foi utilizada a estatística descritiva para a apresentação dos resultados (média \pm desvio padrão). Os dados apresentaram distribuição normal quando verificados utilizando o teste de Shapiro-Wilk. Para as comparações das variáveis foi utilizada ANOVA two-way, seguido por posthoc de Bonferroni quando necessário, assumindo um nível de significância de $p<0,05$.

\section{Resultados}

A frequência cardíaca apresentou comportamento sigmóide na curva FC x carga, como pode ser observado na Figura 1. Em todos os sujeitos os PT foram identificados (Tabela 1). 
No entanto, em treze foram identificados os dois PT - PIFC (64 $\pm 27 \mathrm{~W} ; 126 \pm 12 \mathrm{bpm})$ e PDFC $(177 \pm 45 \mathrm{~W} ; 178 \pm 8 \mathrm{bpm})-$ em conjunto. Neste caso, tanto a carga quanto a $\mathrm{FC}$, em termos absolutos e relativos, foram significativamente maiores no PDFC comparado ao PIFC $(p<0,05)$. Em seis sujeitos foi verificado apenas o PIFC (80 $\pm 32 \mathrm{~W} ; 125 \pm 13 \mathrm{bpm}$ ) e em um sujeito o PDFC (103 W; 150bpm).

Tabela 1. Média e desvio padrão das variáveis identificadas.

\begin{tabular}{|c|c|c|c|c|}
\hline & \multicolumn{2}{|c|}{ PIFC e PDFC } & \multirow{2}{*}{ PIFC } & \multirow{2}{*}{ PDFC } \\
\hline & PIFC & PDFC & & \\
\hline $\mathbf{n}$ & \multicolumn{2}{|c|}{13} & 6 & 1 \\
\hline Wmáx (W) & \multicolumn{2}{|c|}{$218 \pm 51$} & $228 \pm 40$ & 180 \\
\hline Carga (W) & $64 \pm 27$ & $177 \pm 45^{\star}$ & $80 \pm 32$ & 103 \\
\hline \% Wmáx & $29 \pm 9$ & $81 \pm 10^{\star}$ & $36 \pm 14$ & 57 \\
\hline FC (bpm) & $126 \pm 12$ & $178 \pm 8^{*}$ & $125 \pm 13$ & 150 \\
\hline \% FCmáx & $66 \pm 5$ & $93 \pm 4^{*}$ & $66 \pm 5$ & 82 \\
\hline
\end{tabular}

Valores em média \pm DP. n: número de sujeitos; Wmáx: carga máxima; \%Wmáx: percentual da cara máxima; FC: frequência cardíaca; \%FC: percentual da frequência cárdica. * Significativamente maior que o PIFC.

Tabela 2. Comparação entre a carga e FC nos grupos masculino e feminino.

\begin{tabular}{ccccc}
\hline \multirow{2}{*}{ Fcmáx (bpm) } & \multicolumn{2}{c}{ Masculino } & \multicolumn{2}{c}{ Feminino } \\
\cline { 2 - 5 } & Absoluto & Relativo (\%) & Absoluto & Relativo (\%) \\
\cline { 2 - 5 } Wmáx (W) & $190 \pm 7$ & $95,6 \pm 3,4$ & $189 \pm 8$ & $94,8 \pm 4,1$ \\
FC - PIFC (bpm) & $259 \pm 21$ & & $178 \pm 20^{*}$ & \\
FC - PDFC (bpm) & $124 \pm 10$ & $64,9 \pm 5,0$ & $128 \pm 13$ & $67,2 \pm 4,8$ \\
Valores em média + DP. FCmáx: frequência cardíaca máxima; Wmáx: carga máxima; FC - PIFC:
\end{tabular}

Valores em média \pm DP. FCmáx: frequência cardíaca máxima; Wmáx: carga máxima; FC - PIFC frequência cardíaca no PIFC; FC - PDFC: frequência cardíaca no PDFC. 'Significativamente menor que o PDFC. *Significativamente menor que o grupo masculino.

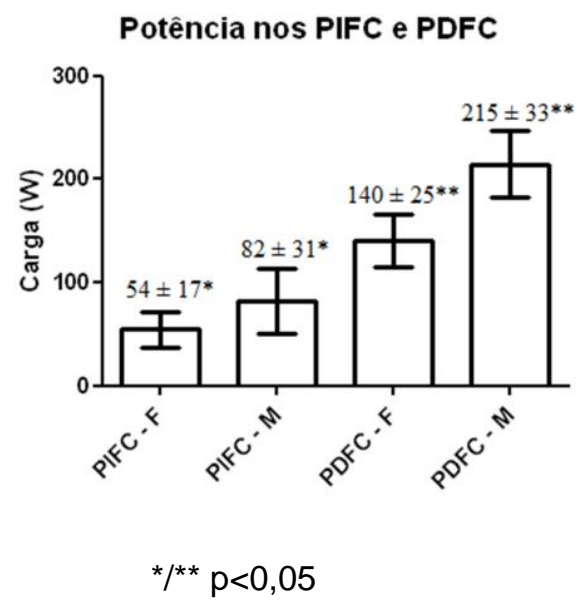

Figura 5. Valores absolutos de carga (W) nos pontos de transição para o grupo feminino (F) e masculino (M). * diferença significativa entre W no PIFC entre os grupos $F$ e M. ** diferença significativa entre a W no PDFC entre os grupos $\mathrm{F}$ e $\mathrm{M}$.

Para verificar a identificação dos PT em sujeitos de ambos os sexos, foram realizadas comparações entre homens e mulheres. O grupo masculino apresentou maior carga máxima que 0 feminino. Os valores de FC nos PT não apresentaram diferença entre os sexos (Tabela 2). O grupo masculino apresentou maiores cargas absolutas tanto para o PIFC quanto para o PDFC do que as mulheres ( $p<0,05$, figura 5 ). No entanto, tanto o PIFC quando o de PDFC quando analisados em termos relativos foram 
encontrados em percentual de carga semelhante $(p>0,05)$, como pode ser verificado na Figura 6 .

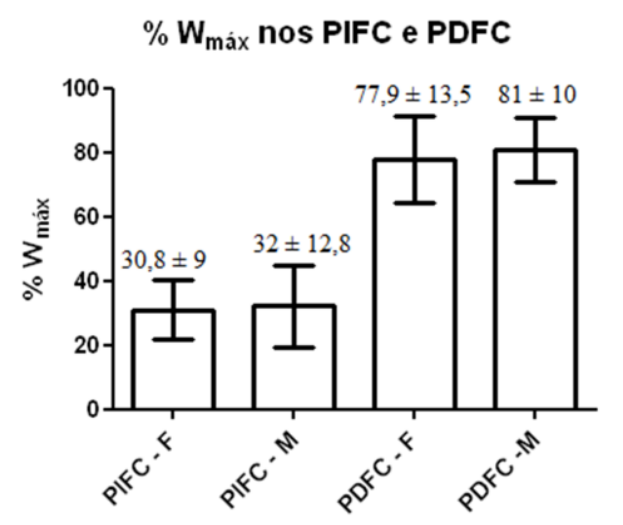

Figura 6. Valores relativos de carga (W) nos pontos de transição para o grupo feminino $(F)$ e masculino (M).

\section{Discussão}

O presente estudo apresenta uma abordagem alternativa para análise dos dados de FC em teste progressivo máximo em cicloergômetro, com evidências de que a utilização dos ajustes polinomial e linear pode contribuir para o estudo e entendimento do comportamento da FC durante o esforço. Do nosso conhecimento, este é o primeiro estudo com a identificação do PIFC e PDFC utilizando o modelo matemático de Cambri et al. (2006), adaptado do modelo Dmáx, em teste progressivo máximo no cicloergômetro, e comparando homens e mulheres.

Os valores de FCmáx encontrados estão em $95 \pm 4 \%$ da FCmáx predita para os avaliados, condição importante para estudos de comportamento da FC e determinação de zonas de transição em distintos pontos da curva.

A FC apresentou comportamento sigmoide e foi possível identificar os PT em todos os avaliados, porém em $35 \%$ deles os pontos não foram encontrados em conjunto (PIFC e PDFC). Diferenças interindividuais podem influenciar nas respostas fisiológicas frente ao exercício físico.

A análise dos dados mostrou que o PIFC foi encontrado a $66 \%$ da FCmáx e $29 \%$ da Pmáx, valor menor que os normalmente encontrados, entre $60 \%$ a $78 \%$ Pmáx (LIMA, 1997; PIOVEZANA; De-OLIVEIRA, 2005) e 67\% do Pico de Velocidade em pista (De-OLIVEIRA, 2004). Já o PDFC foi encontrado a $93 \%$ da FCmáx, valor que corrobora com os encontrados na literatura (BODNER; RHODES, 2000; LIMA, 1997; De-
OLIVEIRA, 2004) e $81 \%$ da potência máxima (Pmáx). Estas discrepâncias podem ser devido às diferenças metodológicas entre os estudos, como a metodologia do teste e de identificação dos PT, e nem todos iniciaram o teste com cargas baixas, analisando toda a curva de FC, além das diferenças no nível de aptidão aeróbia das amostras.

No estudo de Cambri et al. (2006) foi analisado o comportamento da FC em teste progressivo máximo em esteira rolante com cargas de trabalho iniciais de baixa intensidade e utilizando a metodologia matemática de identificação dos PT, observando a existência tanto do PIFC quanto do PDFC em fases iniciais e finais da curva, que apresentou comportamento curvilinear. Os valores relativos de FC no PIFC quando encontrado em fases iniciais foram semelhantes (64,4 $\pm 5,3 \%$ FCmáx), porém os valores de carga de trabalho no PIFC foi superior $(51 \pm 0,5 \%$ do pico de velocidade) comparada ao presente estudo ( $29 \pm 9 \%$ da potência máxima). Pires et al. (2008) encontraram o PIFC entre $28,5 \%$ e $41,9 \%$ da carga máxima (Wmáx), e o PDFC entre $74,4 \%$ e $91,7 \%$ Wmáx, uma vez que utilizaram diferentes métodos de identificação dos PT método visual, Dmáx, logarítmico e $2^{\mathrm{a}}$ derivada em teste incremental $15 \mathrm{~W} / \mathrm{min}$, com carga inicial de $45 \mathrm{~W}$.

Apesar das diferenças de cargas em termos absolutos entre o grupo masculino e o feminino, não houve diferença significativa quando comparados os valores relativos de $\mathrm{FC}$ e potência nos PT (Figuras 5 e 6). A maior carga absoluta nos homens pode ser atribuída a maior força muscular, maior massa magra e fibras musculares mais extensas neste grupo (LEWIS et al., 1986; MILLER et al., 1993). Esses resultados sugerem que a identificação dos PT é capaz de discriminar a capacidade aeróbia e é independente do sexo, pois encontramos intensidades relativas semelhantes para os dois grupos estudados.

O PIFC foi encontrado em cargas significativamente inferiores a do PDFC, tanto para valores absolutos quanto relativos. Ainda que os mecanismos fisiológicos não sejam completamente compreendidos, algumas possibilidades são discutidas na literatura. Alonso et al. (1998) sugerem que no início do exercício progressivo máximo ocorre retirada parassimpática devido à irradiação cortical sobre 
a região bulbar, e incremento da atividade simpática nos estágios subsequentes do teste, para ajustar o sistema cardiovascular à condição imposta. Esses ajustes provocam um comportamento da FC que permite a identificação do PIFC em testes progressivos máximos com baixas cargas iniciais, pequenos incrementos de intensidade e estágios de curta duração.

Para o PDFC, as influências parecem estar ligadas a função do miocárdio, a atividade do sistema neural e o nível de catecolaminas e de potássio $\left(\mathrm{K}^{+}\right)$(IGNJATOVIC et al., 2008). Os nervos cardioaceleradores simpáticos estimulam a liberação das catecolaminas adrenalina e noradrenalina, que provocam aumento dos batimentos cardíacos, pois agem acelerando a despolarização do nódulo sinoatrial, e também provocam um aumento na força de contração do miocárdio, aumentando assim o volume de ejeção do ventrículo esquerdo, que permite que o débito cardíaco se mantenha elevado em altas intensidades sem que ocorram grandes aumentos na FC e provocam o redimensionamento do fluxo sanguíneo para as áreas musculares em atividade (McARDLE et al., 2008; POKAN et al., 1993; POKAN et al., 1995). A ação metabolorreflexa aferente iniciada na musculatura esquelética ativa colabora com estes ajustes cardiovasculares (ALONSO et al. 1998). O aumento da concentração plasmática de potássio $\left(\mathrm{K}^{+}\right)$também faz com que aumente $\mathrm{o}$ volume de ejeção, provocando um aumento menos pronunciado da FC, que então tende a um platô (HOFMANN et al., 1994).

Hofmann et al. (2005) ponderaram que as hipóteses apresentadas acima permitem apenas a descrição do fenômeno e não a explicação de seu mecanismo fisiológico, apresentando a hipótese de que a origem fisiológica do PDFC seja a sensibilidade dos B1-adrenoreceptores. Os adrenoreceptores são uma classe de proteína que são alvos das catecolaminas, especialmente a noradrenalina e adrenalina, que diante da ligação de um agonista geralmente causa uma resposta simpática, com aumento da FC e redimensionamento do fluxo sanguíneo de órgãos não essenciais durante 0 exercício para 0 músculo esquelético.

Sujeitos que apresentam maior sensibilidade dos B1-adrenoreceptores têm resposta regular (sigmóide) da FC, pois apesar dos baixos níveis de catecolaminas em cargas anteriores ao segundo limiar, os B1-adrenoreceptores são estimulados. Assim, em cargas acima do segundo limiar, quando há aumento das catecolaminas, os receptores estão saturados e não ocorrem grandes aumentos da FC, preservando assim a função ventricular esquerda (HOFMANN et al. 2005).

Os exatos mecanismos fisiológicos que permitem a identificação dos PT ainda não estão completamente esclarecidos, entretanto os recentes achados indicam que os PT a partir da FC representam mudanças fisiológicas em relação à demanda do exercício.

A identificação dos PT da FC utilizando o método Dmáx Cambri parece ser objetiva, prática e de baixo custo operacional. No presente estudo foi possível identificar dois pontos de transição em diferentes domínios fisiológicos. Porém, nossos resultados limitam-se por não serem comparados com medidas diretas de lactato sanguíneo e/ou ventilação durante 0 teste progressivo máximo empregado. Os PT a partir de curvas da FC podem ser úteis na prescrição $e$ acompanhamento de treinamento, mas sua aplicação pode ser limitada, pois nem todos os sujeitos apresentam esses fenômenos em conjunto.

\section{Conclusão}

Em teste progressivo máximo no cicloergômetro com baixas cargas iniciais de trabalho a FC apresentou comportamento sigmóide. A carga e a FC nos PT não difere entre sujeitos do sexo masculino e feminino em termos relativos. O PIFC foi encontrado em carga significativamente inferior ao PDFC e parece ser uma variável fisiológica generalizável, sendo que, pelo número de identificações em conjunto (2/3 dos sujeitos) restam dúvidas referentes à determinação dos dois PT.

\section{Referências}

ALONSO, D. O.; FORJAZ, C. L. M.; REZENDE, L. O.; BRAGA, A. M. F. W.; BARETTO, A. C. P.; NEGRÃO, C. E.; RONDON, M. U. P. B.

Comportamento da frequência cardíaca e da sua variabilidade durante as diferentes fases do exercício físico progressivo máximo. Arquivos Brasileiros de Cardiologia, São Paulo, v. 71, n. 6, p. 787-792, 1998.

\section{ASSOCIAÇÃO MÉDICA MUNDIAL. Declaração}

de Helsinki V. Adotada na 18a. Assembléia Médica Mundial, Helsinki, Finlândia (1964), 
alterada na 29a. Assembléia, em Tóquio, Japão (1975), 35a. em Veneza, Itália (1983), 41a. em Hong Kong (1989) e 48a. Sommerset West/África do Sul, 1996. Disponível em: http://www.ghente.org/doc juridicos/helsinki5.htm. Acesso em: 20 nov. 2008

BERNARD, T.; GAVARRY, O.; BERMON, S.; GIACONOMI, M.; MARCONNET, P.;

FALGAIRETTE, G. Relationships between oxygen consumption and heart rate in transitory and steady states of exercise and during recovery: influence of type of exercise. European Journal of Applied Physiology, Berlin, v. 75, p. 170-176, 1997.

BODNER, M. E.; RHODES, E.C. A review of the concept of the heart rate deflection point. Sports Medicine, Auckland, v. 30, n. 1, p. 31-46, Jul. 2000.

BROOKE, J.D.; HAMLEY, E. J. The heart-ratephysical work curve analysis for the prediction of exhausting work ability. Medicine \& Science in Sports \& Exercise, Madison, v. 4, p. 23-6, 1972.

BUNC, V.; HOFMANN, P.; LEITNER, H.; GAISL, $G$. Verification of the heart rate threshold. European Journal of Applied Physiology, Berlin, v. 70, p. 263-269, 1995.

CAMBRI, L. T.; FOZA, V.; NAKAMURA, F. Y.; DeOLIVEIRA, F.R. Frequência cardíaca e a identificação dos pontos de transição metabólica em esteira rolante. Revista da Educação Física, Maringá, v. 17, n. 2, p. 131-137, 2006.

CARMINATTI, L. J. Validade de limiares anaeróbios derivados do teste incremental de corrida intermitente (tcar) como preditores do máximo steady- state de lactato em jogadores de futsal. 2006. Dissertação (Mestrado em Ciências do Movimento Humano, Fisiologia do Movimento) - Centro de Educação Física, Fisioterapia e Desportos, Universidade do Estado de Santa Catarina, Florianópolis, 2006.

CONCONI, F.; FERRARI, M.; ZIGLIO P. G.; DROGHETTI, P.; CODECA, L. Determination of the anaerobic threshold by a noninvasive field test in runners. Journal of Applied Physiology, Bethesda, v. 52, n. 4, p. 869-73, 1982.

CONCONI, F.; GRAZZI, G.; CASONI, I.; GUGLIELMINI, C.; BORSETTO, E.; BALLARIN, G.; et al. The Conconi Test: methodology after 12 years of the application. International Journal of Sports Medicine, Stuttgart, v. 17, p. 509-519, 1996.

De-OLIVEIRA, F. R. Prediccion de los umbrales de lactato y ajustes de frecuencia cardiaca en el test de legerboucher. 2004. Tese (Doutorado em Atividade Física e Esporte) - Facultad de Filosofia, y Ciências de la Educación, San Sebastian, 2004.

FOX, E. L.; BOWERS, R. W. \& FOSS, M. L. Bases Fisiológicas da Educação Física e dos Desportos. Rio de Janeiro: Guanabara Koogan, 1998.

HAWKINS, M. N.; RAVEN, P. B.; SNELL, P. G.; STRAY-GUNDERSEN, J.; LEVINE, B. D. Maximal oxygen uptake as a parametric measure of cardiorespiratory capacity. Medicine \& Science in Sports \& Exercise, Madison, v. 39, n. 1, p. 103-7, 2007.

HOFMANN P.; ' R.; PREIDLER K.; LEITNER H.; SZOLAR D.; EBER B.; SCHABERGER G.

Relationship between heart rate threshold lactate turn point and myocardial function. International Journal of Sports Medicine, Stuttgart, v. 15, n. 5, p. 232-237, 1994.

HOFMANN, P.; WONISCH, M.; POKAN, R.; SCHWABERGER, G.; SMEKAL, G.; DUVILLARD, S. P. $\beta 1$ - Adenoceptor mediated origin of the heart rate performance curve deflection. Medicine \& Science in Sports \& Exercise, Madison, v. 37, n. 10, p. 1704-1709, 2005.

IGNJATOVIĆ, A.; HOFMANN, P.; RADOVANOVIĆ, D. Non-Invasive Determination of the Anaerobic Threshold Based on the Heart Rate Deflection Point. Facta universitatis: Series Physical Education and Sport, Niš, v. 6, n. 1, p. 1-10, 2008.

LEWIS, D.A.; KAMON, E.; HODGSON, J.L. Physiological differences between genders. Implications for sports conditioning. Sports Medicine, Auckland, v. 3, n. 5, p. 357-69, 1986.

LIMA, J. P. Frequência cardíaca em cargas crescentes de trabalho: ajuste sigmóide, ponto de inflexão e limiar de variabilidade da frequência cardíaca. 1997. Tese (Doutorado em Biodinâmica do Movimento Humano) - Escola de Educação Física e Esporte, Universidade de São Paulo, São Paulo, 1997.

LOHMAN, T. G. Skinfolds and body density and their relation of body fatness: A review. Human Biology, Belmont, v. 53, n. 2, p. 181-225, 1981.

LUCIA, A.; CARVAJAL, A.; BORAITA, A.; SERRATOSA L.; HYOS, J.; CHICHARRO J. L. Heart dimensions might influence the occurrence of heart rate deflection point in higly trained cyclists. Brazilian Journal of Sports Medicine, São Paulo, v. 33, p. 387-392 ,1999.

McARDLE, W.D.; KATCH, F.I; KATCH, V.L. Fisiologia do exercício: Energia, nutrição e 
desempenho humano. 6 ed. Tradução de Giuseppe Taranto. Rio de Janeiro: Guanabara Koogan, 2008.

MILLER, A. E. J.; MacDOUGALL, J. D.; TARNOPOLSKY, M. A.; SALE, D. G. Gender differences in strength and muscle fiber characteristics. European Journal of Applied Physiology and Occupational Physiology, Berlin, v. 66, n. 3, p. 254-262, 1993.

PIOVEZANA, P. S.; De-OLIVEIRA, F. R. Reprodutibilidade das variáveis derivadas da curva de frequência cardíaca em teste progressivo. Revista Digital Efdeportes, Buenos Aires, v. 10, n. 90, nov. 2005. Disponível em: http://www.efdeportes.com/efd90/test.htm. Acesso em: 15 jan 2008

PIRES, F. O.; CARMO, I. M. L.; ARROYO, F. N.; De-OLIVEIRA; F. R. Característica sigmóide da FC durante teste progressivo e aplicação de diferentes métodos de identificação dos limiares de FC. Revista Mackenzie de Educação Física e Esporte, São Paulo, v. 7, n. 1, 2008. Disponível em:

http://www3.mackenzie.br/editora/index.php/remef /article/viewFile/1213/904. Acesso em: 12 set. 2008

POKAN, R.; HOFMANN, P.; PREIDLER, K.; LEITNER, H.; DUSLEAG, J.; EBER, B.; et al. Correlation between inflection of the heart rate/work performance curve and myocardial function in exhausting cycling ergometer exercise. European Journal of Applied Physiology and Occupational Physiology, v. 67, n. 5, p. 385388, 1993.

POKAN, R.; HOFMANN, P.; LEHMANN, M.; LEITNER, H.; EBER, B., GASSER R.; SCHWABERGER G.; KEUL J.; KLEIN W. Heart rate deflection related to lactate performance curve and plasma catecholamine response during incremental cycle ergometer exercise. European Journal of Applied Physiology, Berlin, v. 70, p. 175-179, 1995.

RIBEIRO, J.P.; FIELDING R. A.; HUGHES, V.; BLACK, A.; BOCHESE, M. A.; KNUTTGEN, H. G. Heart rate break point may coincide with the anaerobic and not the aerobic threshold. International Journal of Sports Medicine, Stuttgart, v. 6, n. 4, p. 220-4, 1985.

SKINNER, J. S.; McLELLAN, H. The transition from aerobic to anaerobic metabolism. Reseach Quartely for Exercise and Sport, Washington, n. 51, p. 234-48, 1980.

SURAWICZ, B.; PARIKH, S. R. Differences between ventricular repolarization in men and women: description, mechanism and implications.
Annals of Noninvasive Electrocardiology, Armonk, v. 4, n. 8, p. 333-40, 2003.

WILMORE, J. H.; COSTILL, D. L. Fisiologia do esporte e do exercício. São Paulo: Manole, 2001.

Apoio financeiro: Bolsa de estudos PIBICFAPEMIG e CNPq

Agradecimento: À Rafaela F. Villanova e Adriano E. Lima-Silva pelas contribuições na elaboração do estudo.

\section{Endereço:}

Patrícia Guimarães Couto

Escola de Educação Física e Esportes - USP Av. Prof. Mello de Morais, 65 - C. Universitária 05508-030

São Paulo SP Brasil

Telefone: (11) 3091.3095

e-mail: patriciagcouto@usp.br

Recebido em: 7 de abril de 2010.

Aceito em: 7 de abril de 2013.

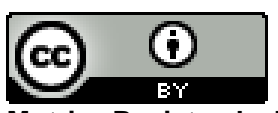

Motriz. Revista de Educação Física. UNESP, Rio Claro, SP, Brasil - elSSN: 1980-6574 - está licenciada sob Creative Commons - Atribuicão 3.0 